\title{
Determining diagnostic markers of vitamin B12 status in older adults- Data from the Trinity Ulster Department of Agriculture Ageing cohort study
}

\author{
E. Laird ${ }^{1}$, M. Ward ${ }^{2}$, H. McNulty ${ }^{2}$, L. Hoey ${ }^{2}$, J. J. Strain ${ }^{2}$, M. C. Casey ${ }^{3}$, C. Cunningham ${ }^{3}$ \\ and A. M. Molloy ${ }^{1}$ \\ ${ }_{1}^{1}$ Institute of Molecular Medicine, School of Medicine, Trinity College, Dublin, ${ }^{2}$ Northern Ireland Centre for Food and \\ Health, University of Ulster, Coleraine, BT52 ISA and ${ }^{3}$ The Mercers Institute for Research on Ageing, \\ St James's Hospital, Dublin
}

Vitamin B12 deficiency is a significant public health issue, particularly within the elderly population with estimates of those affected ranging from $5-40 \%$ depending on the marker of measurement. However, diagnosis is difficult because haematological symptoms of deficiency are often absent and many present with diffuse, non-specific neurological symptoms. Biochemical markers of B-12 status have been previously investigated for their clinical utility, specificity and sensitivity in determining true status; however, few studies have investigated these in large, well-characterised clinically appropriate populations (particularly older adults and those with renal impairment).

Participants ( $n$ 5201) were recruited to the TUDA ageing cohort study from the University of Ulster, Coleraine and those attending the memory and bone clinics in the Geriatric Unit of St. James Hospital, Dublin. Those receiving B12 treatment/supplementation were excluded ( $n$ 521) from the final analysis. Blood samples were analysed for total serum cobalamin, holotranscobalmin (Holo TC), homocysteine (tHcy), red cell folate (RCF) and serum folate at Trinity College Dublin. Methylmalonic acid (MMA) determination was performed on a subset of samples $(n$ 1399) at the University of Bergen, Norway. Hematologic and renal function data were available through the main study database and the estimated glomerular filtration rate (eGFR) was calculated by use of the Cockcroft-Gault equation. A separate reference population of 459 healthy volunteers (228 Male, 231 Female; 18-84yrs) from the National Adult Nutrition Survey (NANs) was used to determine a reference interval for HoloTC (23.4 pmol/1) and for total serum cobalamin $(129 \cdot 1 \mathrm{pmol} / \mathrm{l})$. Vitamin B12 deficiency was defined as a MMA concentration $(>0 \cdot 45 \mu \mathrm{mol} / \mathrm{l})$.

Table 1. Performance of Holo TC \& Serum cobalamin to detect B12 deficiency in eGFR ranges using reference cut-off intervals ${ }^{1}$

\begin{tabular}{|c|c|c|c|c|c|}
\hline eGFR Range (ml/min) & $n$ & Sensitivity, $\%(95 \% \mathrm{CI})$ & Specificity, \% $(95 \% \mathrm{CI})$ & $\mathrm{PPV}^{2}, \%(95 \% \mathrm{CI})$ & $\mathrm{NPV}^{3}, \%(95 \% \mathrm{CI})$ \\
\hline \multicolumn{6}{|l|}{$\geq 60$} \\
\hline$\overline{\text { Holo TC }}$ & 612 & $52 \cdot 91(45 \cdot 1-60 \cdot 5)$ & $88 \cdot 44(85 \cdot 0-91 \cdot 2)$ & $64 \cdot 08(55 \cdot 6-71 \cdot 9)$ & $82 \cdot 80(79 \cdot 0-86 \cdot 1)$ \\
\hline Serum Cobalamin & 612 & $39 \cdot 53(32 \cdot 1-47 \cdot 2)$ & $70 \cdot 45(65 \cdot 9-74 \cdot 6)$ & $34 \cdot 34(27 \cdot 7-41 \cdot 4)$ & $74 \cdot 88(70 \cdot 4-78 \cdot 9)$ \\
\hline \multicolumn{6}{|l|}{$30-59$} \\
\hline Holo TC & 635 & $40 \cdot 09(33 \cdot 5-46 \cdot 9)$ & $95 \cdot 45(92 \cdot 9-97 \cdot 2)$ & $82.08(73 \cdot 4-88 \cdot 8)$ & $75 \cdot 43(71 \cdot 5-79 \cdot 0)$ \\
\hline Serum Cobalamin & 628 & $32 \cdot 26(26 \cdot 0-38 \cdot 9)$ & $70 \cdot 45(65 \cdot 9-74 \cdot 6)$ & $55 \cdot 12(46 \cdot 0-63 \cdot 9)$ & $70 \cdot 6(66 \cdot 4-74 \cdot 6)$ \\
\hline \multicolumn{6}{|l|}{$<29$} \\
\hline Holo TC & 133 & $30 \cdot 16(19 \cdot 2-43 \cdot 0)$ & $95 \cdot 71(87 \cdot 9-99 \cdot 0)$ & $86 \cdot 36(65 \cdot 0-96 \cdot 9)$ & $60 \cdot 36(50 \cdot 6-69 \cdot 5)$ \\
\hline Serum Cobalamin & 133 & $17 \cdot 74(9 \cdot 2-29 \cdot 5)$ & $95 \cdot 59(87 \cdot 6-99 \cdot 0)$ & $78 \cdot 57(49 \cdot 2-95 \cdot 0)$ & $56 \cdot 03(46 \cdot 5-65 \cdot 2)$ \\
\hline
\end{tabular}

${ }^{1}$ Reference cut-off from NANs study; ${ }^{2}$ Positive predictive value; ${ }^{3}$ Negative predictive value.

In a ROC plot analysis (with MMA $>0.45 \mu \mathrm{mol} / \mathrm{L}$ classified as B12 deficient status), the areas under the curve (AUCs) demonstrated that Holo TC wasthe best-performing indicator of deficient status (AUC 0.78; 95\% CI 0.76-0.81). The differences in AUC between HoloTC and serum cobalamin $(0 \cdot 64 ; 0 \cdot 61-0 \cdot 67)$, serum folate $(0 \cdot 53 ; 0 \cdot 50-0 \cdot 57)$, RCF $(0 \cdot 58 ; 0.53-0.62)$ and GFR $(0 \cdot 59 ; 0 \cdot 56-0 \cdot 62)$ were significant $(P<0 \cdot 0001)$. These findings support the use of Holo TC as a first line diagnostic marker for determination of B12 status in older adults, including those with renal impairment.

Acknowledgement of Funding: The Irish Department of Agriculture, Food and the Marine (DAFF) and the Northern Ireland Department for Employment \& Learning (DEL) through its "Strengthening the All-Island Research Base" initiative. Funding of Holo TC and MMA analysis: Axis Shield Diagnostics, Dundee, Scotland. 